\title{
Human learning follows the dynamics of gradient descent
}

2

3
Daniel N. Barry ${ }^{1}$ and Bradley C. Love ${ }^{1,2}$

1. Department of Experimental Psychology, University College London, 26 Bedford Way, London, WC1H0AP, UK

2. The Alan Turing Institute, 96 Euston Road, London, NW12DB, UK Corresponding author: daniel.barry@ucl.ac.uk, ORCID ID: 0000-0002-2474-5651

Word Count: 2986

\section{8}

9

1

2

4

5

16


Artificial neural networks (ANNs) have achieved near human-level performance on many tasks and can account for human behavioural and brain measures in a number of domains. Although a principal strength of ANNs is learning representations from experience, only a handful of contributions have evaluated this process to ask whether ANN learning dynamics provide a good model of human learning. We investigated whether humans learn similarly to an ANN, which adjusts its representations through gradient descent. Gradient descent learning is steep at first and initially ignores covariance between features. ANNs can theoretically display a nonmonotonic behaviour in which early in learning, multiple weak predictors determine the ANN's decision whereas late in learning a single strong predictor can dominate. This initial behaviour was confirmed in a simple ANN and in half of human participants performing a comparable task. Later in gradient descent learning, the ANN changed to placing a greater weight on the stronger predictor, and humans also shifted their preferences in the same way. Hidden Markov modelling of the behaviour of ANNs and humans predicted similar transitions from weakfeature to strong-feature states. Our results suggest a significant proportion of people learn about categories in a manner analogous to ANNs, possibly by updating their mental representations by a process akin to gradient descent. Our findings demonstrate how ANNs can be used to not only explain the products of human learning but also the process. 


\section{1. Introduction}

53 Artificial neural networks display human-like capabilities on tasks such as object recognition 54 (He, Zhang, Ren, \& Sun, 2015; Russakovsky et al., 2015), speech processing (Graves, 55 Mohamed, \& Hinton, 2013; Kell, Yamins, Shook, Norman-Haignere, \& McDermott, 2018), and 56 game-playing (Badia et al., 2020; Silver et al., 2018). An outstanding question is whether

57 humans update their representations using ANN-like dynamics. Comparisons with human 58 development suggest parallels. Similar to infants, deep ANNs first learn broad conceptual relationships in hierarchically-structured data (Saxe, McClelland, \& Ganguli, 2019). Neural networks also become rapidly sensitive to useful inputs, yielding sudden boosts in performance, and this signature of gradient descent can be observed in comparable stagelike progress observed in children (Munakata \& McClelland, 2003). Such non-monotonic learning curves also emerge on a feature level in ANNs, predicting the illusory correlations children often believe are present during category learning (Saxe et al., 2019). Further, learning updates in ANNs and humans can be similarly sensitive to order effects, such as those related to overall category variability (Mareschal, Quinn, \& French, 2002) with estimates of variability shaped by trial-to-trial error correction (Sakamoto, Jones, \& Love, 2008). Neural network dynamics may therefore contribute to cognitive science by generating predictions about human learning.

Category learning is a common task for both humans and ANNs, providing a wellcontrolled test bed in which we can examine the dynamics of learning, absent prior knowledge. (Nosofsky \& Palmeri, 1998). However, additional category dimensions, whether relevant or not, dilutes human's reliance on individual features, suggesting people favour using multiple cues (Edgell et al., 1996). Evidence supporting either behaviour is mixed. Gluck, Shohamy, and Myers (2002) showed individuals initially rely on one cue in the weather prediction task, 
before using more later. Alternatively, Lagnado, Newell, Kahan, and Shanks (2006) reported most people base their decisions on multiple cues from the beginning. In this study, will make a neural network-based prediction on how humans solve a categorisation task with multiple features.

We predict ANNs will rely on multiple cues early in training, even when doing so is suboptimal. The predominant mechanism underlying neural network learning is stochastic gradient descent (Rumelhart, Hinton, \& Williams, 1986). The gradient informs how a model's weights should change to minimise the difference between its predictions and the data. Weights are changed gradually through descending the error surface (Figure 1B) until optimal values are found. Although the weights in a one-layer network will eventually converge to those from a standard linear regression, gradient descent's incremental nature makes it unlikely that the network will take the most direct route. For example, if similar weight changes are initially applied to all predictive features, the combined weights of multiple weakly predictive features may overshadow a single more useful feature. Although this problem may be exacerbated in a complex non-linear multi-layered network, in principle it should also affect a simple one-layer linear network. We investigated this phenomenon by training a small ANN with three input features, two less predictive than the third, and monitored how the network updates its representations. Using the same design, we compared how human participants learned. We hypothesised both ANNs and human participants would initially assign a higher importance to multiple less predictive features, but this preference would shift towards a stronger feature throughout training.

\section{Materials and methods}

\subsection{Neural networks}

\subsubsection{Zero-initialised neural network and linear regression}

The ANN contained three inputs and one output (Figure 1A). We trained the network on three binary predictor variables, two of which were weaker predictors than the third, and one binary 
A

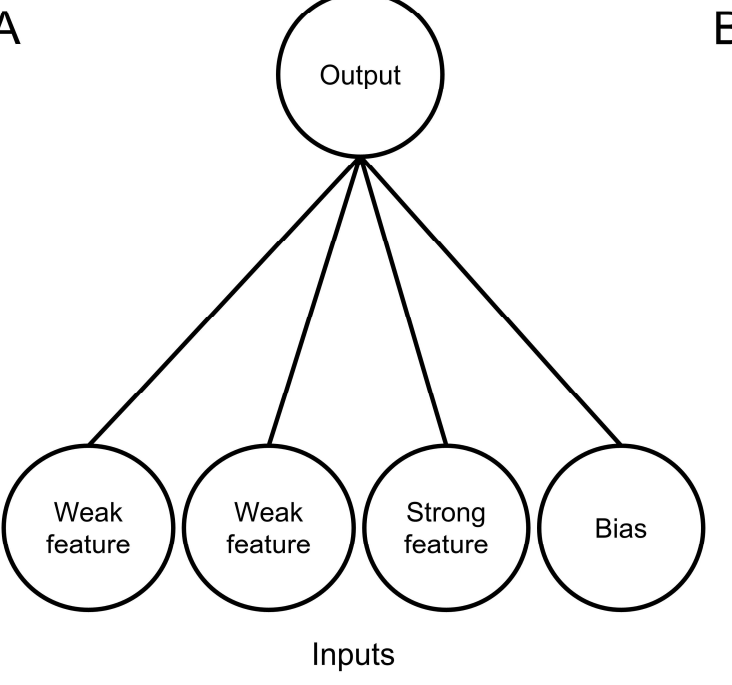

B

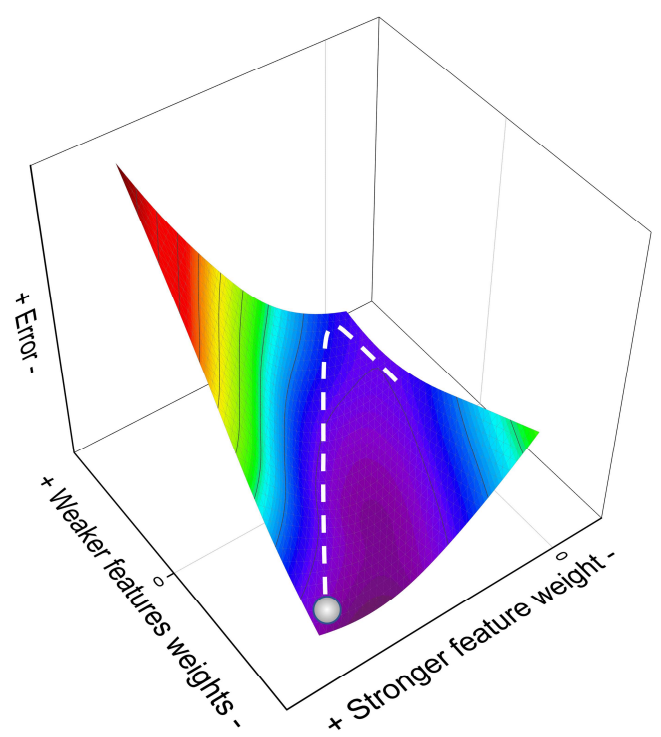


Figure 1. Neural network structure and hypothesised gradient descent trajectory. (A) A simple ANN was trained to predict one outcome variable from three input features, one more strongly predictive than the other two. (B) The hypothesised trajectory of gradient descent along the error surface of the model.

\subsubsection{Randomly initialised neural networks}

To compare neural network behaviour more closely with a sample of human participants, we additionally trained 102 randomly initialised networks (to match the number of human participants, see section 2.2.1), and added noise (mean: 0, SD: 0.1) to the learned weights.

\subsubsection{Analysis}

\subsubsection{Proportion of neural networks using different features for categorisation}

We calculated the proportion of the randomly initialised networks relying more on the combined weaker weights, or a single stronger weight for each of the 4000 blocks.

\subsubsection{Hidden Markov modelling}

To investigate how the randomly initialised networks updated their representations, we obtained the network's decisions based on the feature weights and fitted a Hidden Markov Model (HMM). Here, the objective is to determine the state which is producing a behaviour. However, the state is not directly observable and must be inferred from a sequence. Using the

145 Expectation-Maximisation algorithm, we searched for the maximum likelihood of a number of parameter estimates. These were the probability of transitioning from one state to another from time $t$ to $t+1$, generating a state transition matrix, and the probability of emitting a symbol in that state, known as emission probabilities. As the data involved a binary decision, we used a multinomial HMM using the hmmlearn toolbox (https://github.com/hmmlearn/hmmlearn). We constrained the ANN and human analysis to have the same number of states (see section

151 2.3). To facilitate interpretation, we assigned labels to each of the states based on the 152 emissions probabilities. 


\section{$153 \quad 2.2$ Human category learning task}

\section{$154 \quad$ 2.2.1 Participants}

155 One hundred seventy-four participants were recruited via the online crowdsourcing platform

156 MTurk (www.mturk.com). Informed consent was obtained prior to experimentation. The task was hosted on the online platform Gorilla (https://app.gorilla.sc/). Seventy-two participants failed to learn the task and were excluded (see section 2.3.2). The average age of the remaining 102 participants was 36 (SD 10). Fifty-two participants identified as female and 48 as male.

\subsubsection{Training}

The three binary predictor variables were features of bugs, namely antennae, mouth, and legs (Figure 2B). Each bug belonged to one of two categories. Category labels were generated by belong to either category. the pseudoword generator "Wuggy" (Keuleers \& Brysbaert, 2010). The associations between bug features and labels matched those used during ANN training (Figure 2A). Participants viewed 14 bugs consecutively; each appeared on screen followed by its label (Figure 2C). The assignment of bug features to being the stronger or one of the weaker predictors was counterbalanced across participants, as were six different sets of labels.

Participants underwent 10 training blocks with the same 14 images presented in a random order. After each block, we tested whether participants had learned the basic associations between features and their probabilistic outcomes. In these two "prototype" trials, all three features were predictive of the same category label, and if participants were not responding correctly on these final four trials, they were excluded from the analysis. Of primary interest was the remaining participant's performance on two "conflict" trials, where one feature strongly predicted one category, while the other two weakly predicted another (Figure 2D). In the four test trials participants were asked to rate on a sliding scale how likely the bug was to 179 
Legs Antennae Mouth

(weak) (weak) (strong)
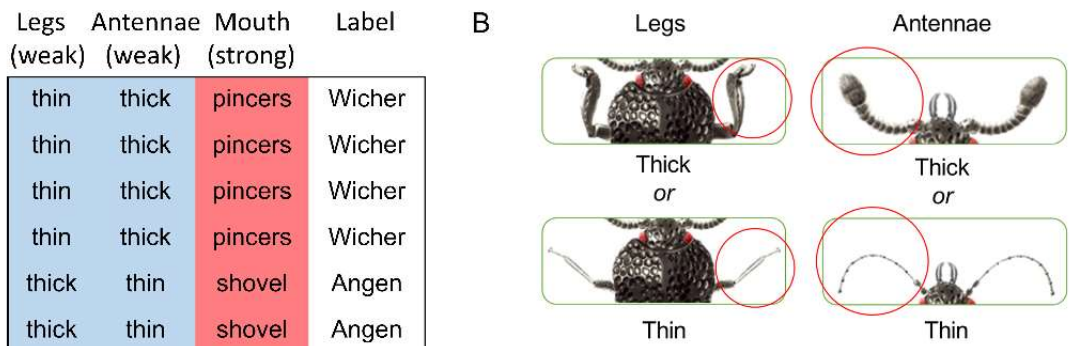

Thick
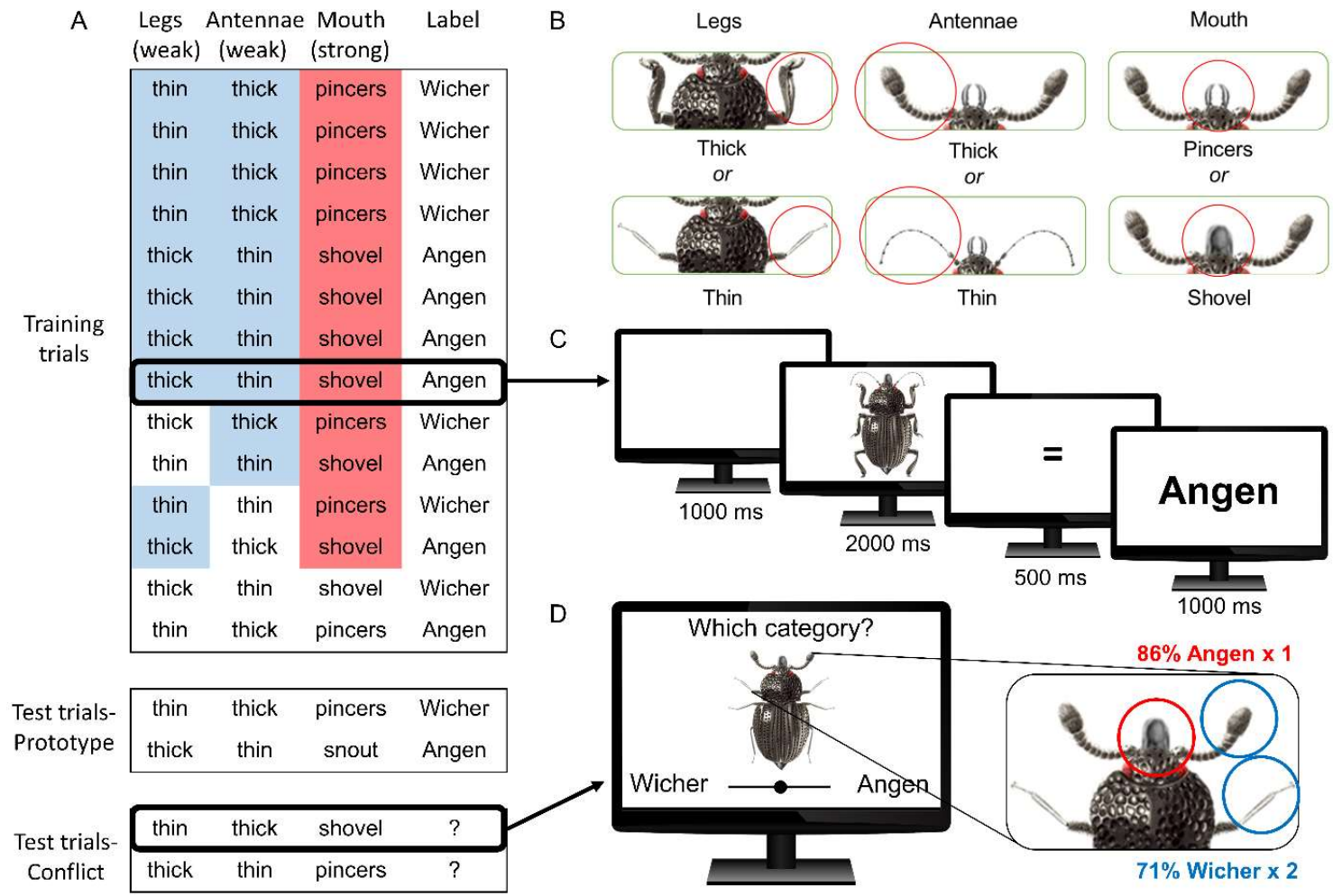

Pincers

Figure 2. The human category learning task. (A) During training, participants were shown

14 bug images, one at a time, which contained a combination of three features. Two of the three features were less predictive of the bug's category (shaded blue-10/14 trials) than the third (shaded red-12/14 trials). During testing, participants were asked to identify the category of prototype bugs, where each feature was predictive of a single category, and conflict bugs, where features predicted different categories. (B) The three bug features which could each differ in two ways: a thick or thin antennae, a pincer or shovel mouth, and thick or thin legs. (C) Participants viewed each bug image followed by its label. (D) A sample conflict trial, where one feature (mouth) strongly predicted the category "Angen", whereas the two other features (antennae and legs) were weaker predictors of the category "Wicher".

\subsubsection{Proportion of participants using different features for categorisation}


195 To investigate whether people relied on a single "stronger" feature to classify an object, or

196 multiple "weaker" features, and how this changed across learning, we recoded participant's 197 rated probabilities of category membership on each conflict trial into a binary decision, and 198 calculated the proportion of participants using the different features.

\subsubsection{Hidden Markov modelling}

To further investigate how participants updated their learning representations, we modelled their category decisions using a Hidden Markov Model (HMM). To determine the number of states which explained our data we calculated the Bayesian Information Criterion (BIC) for models containing two to eight states. BIC is based on the likelihood of each model, but penalises complex models containing more free parameters. The model with the lowest BIC value was one with three states (see supplementary material). We fitted a three-state HMM to the 20 conflict trials across 102 participants.

\section{Results}

\section{0}

\subsection{Evolution of feature weights during gradient descent learning}

Our simulation investigated how gradient descent assigns weights to different features over the course of learning in an ANN. Each item had three binary predictors (plus a bias term) and a binary outcome variable. The linear regression solution was a weight of 1.0 for the stronger feature and -.2 for each weaker feature (Figure $3 \mathrm{~A}$ ). While gradient descent converged to the same solution, it followed a path in which the weaker features dominated early in learning with the optimal strong feature emphasised later in learning. This simulation suggests that gradient descent may be biased towards abundant but poorly predictive features early in training, before the optimal solution is found. A sample of 102 randomly initialised neural networks performing the same task, which attempted to predict the behaviour of a group of humans, revealed that approximately half of networks began using the weaker cues, slowly transitioning 
222 half of the sample of neural networks begin training highly confident about the importance of 223 the weaker weights (Figure 3C). The model predicted a gradual sequential transition to two 224 "strong feature" states, the first unsure, and the final state highly confident.

A

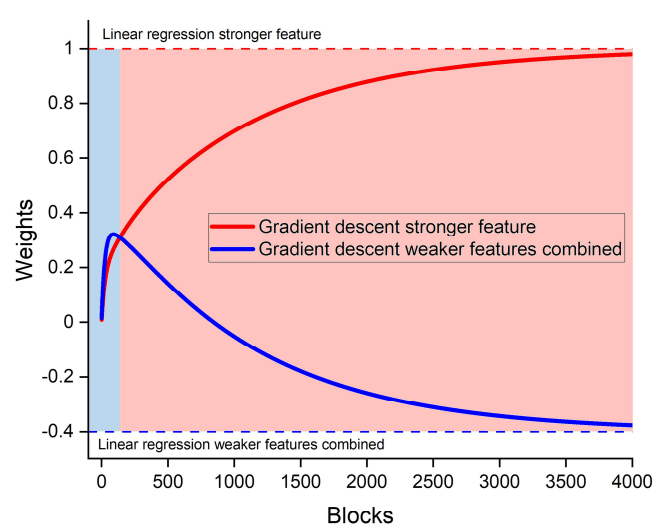

B

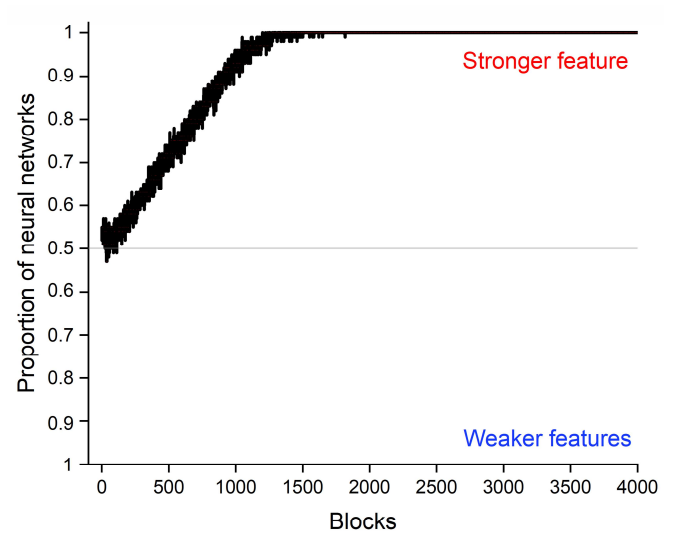

C

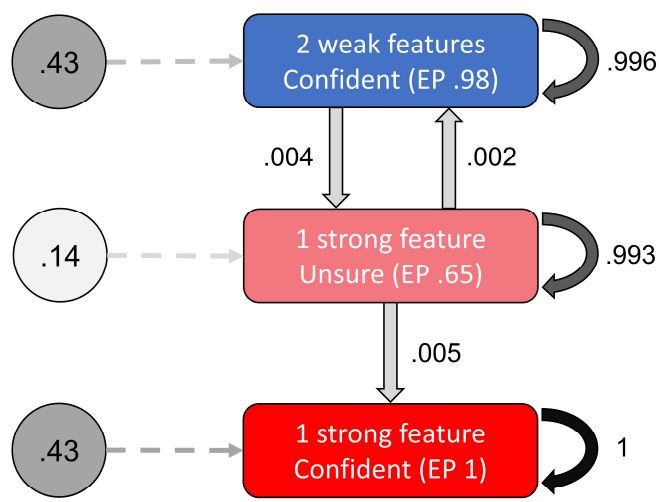

Starting probabilities
Hidden state emission \& transition probabilities time $\mathrm{t} \rightarrow \mathrm{t}+1$
226

Figure 3. Dynamic changes in feature weights during gradient descent in neural networks. (A) The weights assigned by a zero-initialised ANN to two weakly predictive features or one strongly predictive feature throughout training, compared with the weights of a linear regression. (B) The proportion of a sample of 102 randomly initialised neural networks placing greater weights on combined weaker features or a single stronger one throughout training. (C) A three-state hidden Markov model of the randomly initialised neural network behaviour. $(E P=$ emission probability, transition probabilities between and within states displayed adjacent to arrows. Transition probabilities less than 0.0001 are not displayed)

\subsection{Human reliance on category features throughout learning}


237 We then investigated whether people update their representations like ANNs when faced with

238 a comparable learning problem. Figure 4A shows the proportion of participants who assigned

239 a greater importance to the stronger feature, or the two weaker features, across the 20 conflict

240 trials. Participants were equally likely to rely on the strong or weak features at the beginning

241 of training, however an increasing proportion of participants chose the category associated

242 with the stronger feature as training progressed. This gradual shift on a group level closely

243 resembled the behaviour of a sample of randomly initialised ANNs.

244 We fit a HMM to participant's sequences of decisions. Three hidden states emerged

245 from this analysis (Figure 4B). Similar to the sample of ANNs, a significant proportion (36\%)

246 of people were highly confident that the two weaker features were more predictive at the

247 beginning of training. Also reflective of the shift from two-weak to one-strong predictor in

248 ANNs, these participants were likely to transition to a state where they chose the strong feature

249 most of the time. As with the ANN HMM, the human HMM predicted an ultimate transition to

250 a state where they were highly confident about the predictive ability of the strong feature, and

251 would remain in that state.

252

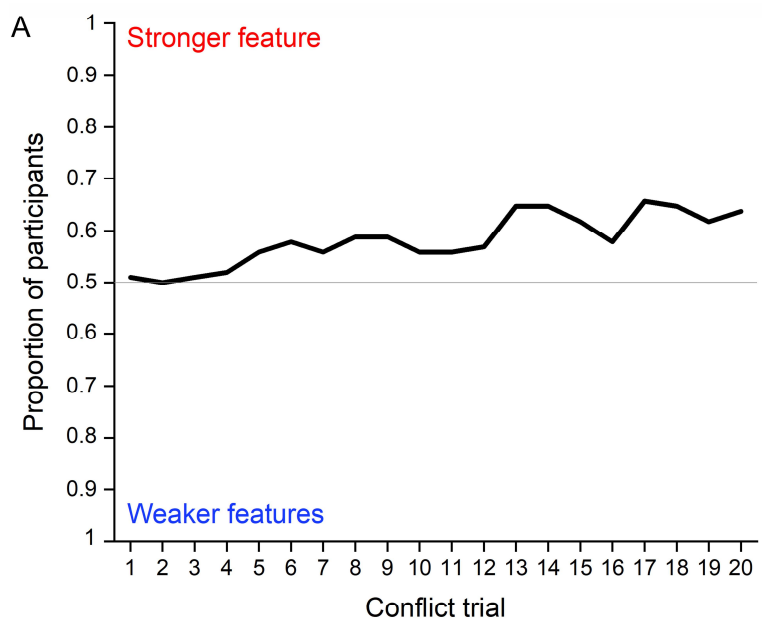

B

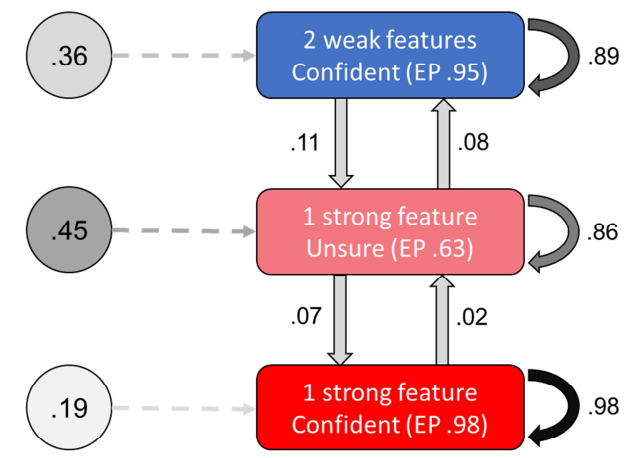

Starting Hidden state emission \& probabilities transition probabilities time $\mathrm{t} \rightarrow \mathrm{t}+1$

254 Figure 4. Individual differences in behaviour throughout human category learning. (A)

255 The proportion of human participants throughout training who chose to classify stimuli based

256 on either one strongly predictive cue or two cues which were more weak predictors. 
257 (B) A HMM fit to participant's responses revealed three states influencing their decisions. (EP

$258=$ emission probability, transition probabilities between and within states displayed adjacent to arrows. Transition probabilities less than 0.0001 are not displayed)

260

\section{Discussion}

We found that the category learning behaviour of human participants could be predicted by the gradient descent updates of randomly initialised simple ANNs performing a similar task. In both cases there was a sub-optimal tendency to begin by relying on multiple weakly predictive cues. Further, humans and ANNs were inclined to change their mind during training and shift their attention towards a stronger feature. This suggests people may share learning mechanisms which mirror those of gradient descent, assigning similar weighting to all predictive features initially and treating them as cumulatively diagnostic of a category. Our results offer a potential mechanistic explanation for previous work in humans demonstrating that the presence of multiple, even redundant cues, can dilute the reliance on stronger ones

271 (Edgell et al., 1996). In the same vein, our results posit a mechanism through which humans and ANNs are vulnerable to being misled by shortcuts, features which are more abundant or obvious at first, but which are not the best predictors of a category (Dagaev et al., 2021). We note that the magnitude of the weight differences in an ANN can be modest at the beginning of training, but we show in a group of neural networks that this would be sufficient to affect behaviour, and reflect that observed in a human sample. While some reservations remain about whether the brain performs computations exactly like gradient descent via backpropagation (Lillicrap, Santoro, Marris, Akerman, \& Hinton, 2020), ANNs which learn this way predict visual brain responses to objects better than any other biologically-inspired models (Cadieu et al., 2014).

We observed in both humans and a group of randomly-initialised ANNs that the shift from a focus on multiple cues to a single cue was gradual, with many people were still relying on the weak cues at the end of training, and many neural networks were still using them after 
1000 blocks. This slow learning may be interpreted as "blocking"; the resistance to learning about a new cue when another has been learned previously (Rescorla \& Wagner, 1972). This has previously been regarded as a product of error-driven learning. However, our results may reveal another significant influence on this phenomenon, that there may be a human analogue of random weight initialisation, whereby pre-existing random biases towards a feature may slow down the updating of category representations.

Prior work using the weather prediction task has been equivocal regarding whether the majority of participants use multiple cues (Lagnado et al., 2006) or a single cue (Gluck et al., 2002) early in category learning. Our task differed from the classic weather prediction task, in that relying on a single strong cue was most efficient, providing a more stringent test of whether people naturally tend towards incorporating multiple cues into their representations. Despite the latter behaviour being sub-optimal, $49 \%$ of participants began training in this manner, $36 \%$ acting with high confidence. These results reveal substantial individual differences across individuals, with some people naturally inclined to adopt a RULEX approach, using a single dimension to classify an object and only adding more if necessary (Nosofsky \& Palmeri, 1998), and others tending to combine across multiple features. Our study has advantages over prior work, benefitting from a larger sample size $(n=102)$, than the Gluck et al. (2002) $(n=30)$ and Lagnado et al. (2006) $(n=12-16)$ studies, yielding more generalisable results. Further, the integrated realistic stimuli we used reflect the naturalistic way humans learn about multidimensional categories (Mack, Love, \& Preston, 2016), in contrast to the separate abstract patterns used in the weather prediction task.

We acknowledge that this study did not evaluate complex tasks, or use a model which builds complex category representations, such as a clustering model (Love \& Medin, 1998). However, this work represents an initial exploration into how people and ANNs can share similar learning dynamics. We hope our results complement prior work, which has revealed similar learning behaviours (Mareschal et al., 2002; Munakata \& McClelland, 2003; Saxe et al., 2019) and neural representations (Güçlü \& van Gerven, 2015; Khaligh-Razavi \& Kriegeskorte, 2014) across humans and ANNs. Neural networks therefore serve to provide 
312 both a mechanistic account and generate specific, and perhaps unexpected, predictions about

313 human behaviour, which might not be compatible with a rational account (Sakamoto et al.,

314 2008), and might not apply to everyone. The human-like behaviour we observed in our simple

315 ANNs may also be consequential for the design of autonomous artificially intelligent agents

316 who engage in continual learning, as they may make sub-optimal decisions early in learning

317 due to the tendency to assign a greater combined importance to less predictive cues.

318 Therefore, although the accuracy of fully trained ANNs can be impressively high (Schrimpf et

319 al., 2018), they may make mistakes similar to those made by humans as they learn about the 320 world.

321

\section{Funding}

323 This work was supported by the Royal Society (grant number 183029); and the Wellcome 324 Trust (grant number WT106931MA) to B.C.L. The funders had no role in study design, data 325 collection and analysis, decision to publish, or preparation of the manuscript.

327 Author Contributions: D.N.B: Conceptualization, methodology, software, data curation, 328 investigation, formal analysis, visualization, writing-original draft preparation, writing-review \& 329 editing. B.C.L.: Conceptualization, methodology, resources, funding acquisition, supervision, 330 writing-review \& editing.

Declaration of interest: None.

Data and code availability: The code and environment required to train the ANN and run the HMM are available at:

https://github.com/danielbarry1/human learning gradient descent

337 All data in the paper, as well as experimental stimuli and instructions, are available at 338 https://doi.org/10.6084/m9.figshare.16930717. 


\section{References}

Badia, A. P., Piot, B., Kapturowski, S., Sprechmann, P., Vitvitskyi, A., Guo, Z. D., \& Blundell, C. (2020). Agent57: Outperforming the atari human benchmark. Paper presented at the International Conference on Machine Learning.

Cadieu, C. F., Hong, H., Yamins, D. L. K., Pinto, N., Ardila, D., Solomon, E. A., ... DiCarlo, J. J. (2014). Deep Neural Networks Rival the Representation of Primate IT Cortex for Core Visual Object Recognition. PLoS Comput Biol, 10(12), e1003963. doi:https://doi.org/10.1371/journal.pcbi.1003963

Dagaev, N., Roads, B. D., Luo, X., Barry, D. N., Patil, K. R., \& Love, B. C. (2021). A Too-Good-to-be-True Prior to Reduce Shortcut Reliance. arXiv preprint arXiv:2102.06406.

Edgell, S. E., Castellan Jr, N. J., Roe, R. M., Barnes, J. M., Ng, P. C., Bright, R. D., \& Ford, L. A. (1996). Irrelevant information in probabilistic categorization. Journal of Experimental Psychology: Learning, Memory, and Cognition, 22(6), 1463-1481. doi:https://doi.org/10.1037/02787393.22.6.1463

Gluck, M. A., Shohamy, D., \& Myers, C. (2002). How do people solve the "weather prediction" task?: individual variability in strategies for probabilistic category learning. Learning \& memory (Cold Spring Harbor, N.Y.), 9(6), 408-418. doi:https://doi.org/10.1101/lm.45202

Graves, A., Mohamed, A.-r., \& Hinton, G. (2013). Speech recognition with deep recurrent neural networks. Paper presented at the 2013 IEEE international conference on acoustics, speech and signal processing.

Güçlü, U., \& van Gerven, M. A. (2015). Deep neural networks reveal a gradient in the complexity of neural representations across the ventral stream. Journal of Neuroscience, 35(27), 1000510014. doi:https://doi.org/10.1523/JNEUROSCI.5023-14.2015

He, K., Zhang, X., Ren, S., \& Sun, J. (2015). Delving deep into rectifiers: Surpassing human-level performance on imagenet classification. Paper presented at the Proceedings of the IEEE international conference on computer vision.

Kell, A. J. E., Yamins, D. L. K., Shook, E. N., Norman-Haignere, S. V., \& McDermott, J. H. (2018). A TaskOptimized Neural Network Replicates Human Auditory Behavior, Predicts Brain Responses, and Reveals a Cortical Processing Hierarchy. Neuron, 98(3), 630-644.e616. doi:https://doi.org/10.1016/j.neuron.2018.03.044

Keuleers, E., \& Brysbaert, M. (2010). Wuggy: A multilingual pseudoword generator. Behavior Research Methods, 42(3), 627-633. doi:https://doi.org/10.3758/BRM.42.3.627

Khaligh-Razavi, S.-M., \& Kriegeskorte, N. (2014). Deep Supervised, but Not Unsupervised, Models May Explain IT Cortical Representation. PLoS Comput Biol, 10(11), e1003915. doi:https://doi.org/10.1371/journal.pcbi.1003915

Lagnado, D. A., Newell, B. R., Kahan, S., \& Shanks, D. R. (2006). Insight and strategy in multiple-cue learning. J Exp Psychol Gen, 135(2), 162-183. doi:https://doi.org/10.1037/00963445.135.2.162

Lillicrap, T. P., Santoro, A., Marris, L., Akerman, C. J., \& Hinton, G. (2020). Backpropagation and the brain. Nat Rev Neurosci, 21(6), 335-346. doi:https://doi.org/10.1038/s41583-020-0277-3

Love, B. C., \& Medin, D. L. (1998). SUSTAIN: A model of human category learning. Aaai/iaai, 671-676.

Mack, M. L., Love, B. C., \& Preston, A. R. (2016). Dynamic updating of hippocampal object representations reflects new conceptual knowledge. Proceedings of the National Academy of Sciences, 113(46), 13203-13208. doi:https://doi.org/10.1073/pnas.1614048113

Mareschal, D., Quinn, P. C., \& French, R. M. (2002). Asymmetric interference in 3- to 4-month-olds' sequential category learning. Cogn Sci, 26(3), 377-389. doi:https://doi.org/10.1207/s15516709cog2603 8

Munakata, Y., \& McClelland, J. L. (2003). Connectionist models of development. Dev Sci, 6(4), 413429. doi:https://doi.org/10.1111/1467-7687.00296 
Nosofsky, R. M., \& Palmeri, T. J. (1998). A rule-plus-exception model for classifying objects in continuous-dimension spaces. Psychon Bull Rev, 5(3), 345-369. doi:https://doi.org/10.3758/BF03208813

Rescorla, R., \& Wagner, A. (1972). A theory of Pavlovian conditioning: The effectiveness of reinforcement and non-reinforcement. Classical Conditioning: Current Research and Theory.

Rumelhart, D. E., Hinton, G. E., \& Williams, R. J. (1986). Learning representations by backpropagating errors. Nature, 323(6088), 533-536. doi:https://doi.org/10.1038/323533a0

Russakovsky, O., Deng, J., Su, H., Krause, J., Satheesh, S., Ma, S., . . Bernstein, M. (2015). Imagenet large scale visual recognition challenge. International Journal of Computer Vision, 115(3), 211-252.

Sakamoto, Y., Jones, M., \& Love, B. C. (2008). Putting the psychology back into psychological models: Mechanistic versus rational approaches. Mem Cognit, 36(6), 1057-1065. doi:https://doi.org/10.3758/MC.36.6.1057

Saxe, A. M., McClelland, J. L., \& Ganguli, S. (2019). A mathematical theory of semantic development in deep neural networks. Proceedings of the National Academy of Sciences, 116(23), 1153711546. doi:https://doi.org/10.1073/pnas.1820226116

Schrimpf, M., Kubilius, J., Hong, H., Majaj, N. J., Rajalingham, R., Issa, E. B., . . DiCarlo, J. J. (2018). Brain-Score: Which artificial neural network for object recognition is most brain-Like? bioRxiv, 407007. doi:https://doi.org/10.1101/407007

Silver, D., Hubert, T., Schrittwieser, J., Antonoglou, I., Lai, M., Guez, A., . . Hassabis, D. (2018). A general reinforcement learning algorithm that masters chess, shogi, and Go through selfplay. Science, 362(6419), 1140-1144. doi:https://doi.org/10.1126/science.aar6404 